\section{Design and Synthesis of Novel Quinazoline Derivatives and Their Evaluation as PI3Ks Inhibitors}

\author{
Omar Maged El-Said, ${ }^{a}$ Mostafa Mohamed Hamed, ${ }^{a, b}$ \\ Stefan Laufer, ${ }^{c}$ and Ashraf Hassan Abadi*,a
}

${ }^{a}$ Department of Pharmaceutical Chemistry, Faculty of Pharmacy and Biotechnology, German University in Cairo; Cairo 11835, Egypt: ${ }^{b}$ Helmholz Institute for Pharmaceutical Research, Saarland University; Campus C2.3, D-66123 Saarbrücken, Germany: and ${ }^{c}$ Department of Pharmaceutical \& Medicinal Chemistry, Institute of Pharmacy, Eberhard-Karls-University Tuebingen; Auf der Morgenstelle 8, 72076 Tübingen, Germany. Received August 3, 2014; accepted September 7, 2014

The aim of this work was to synthesize 4-acetamido-, 4-amino- and 4-oxo-6-substituted aminoquinazolines and to evaluate them as phosphoinositide 3-kinases (PI3Ks) inhibitors. The respective chemotype was designed based on combining the structural features of two previously reported scaffolds acting as potent PI3K $\gamma$ inhibitors, which are quinazoline derivatives and amino-heterocyclic derivatives. In vitro enzymatic assay at $10 \mu \mathrm{M}$ against all the eight human PI3K isoforms showed that an unsubstituted benzamide group at position 6 and an acetyl group at $N^{4}$ gave the best inhibitory activity on PI3K $\gamma$. Interestingly, compounds 5a and 5e showed a significant, inhibitory effect on Class II PI3K-C $2 \gamma$. This is of high value since there are very few inhibitors for this isoform reported in the literature.

Key words kinase; phosphoinositide; inhibitor; aminoquinazoline

Phosphoinositide 3-kinases (PI3Ks) are a family of lipid kinases that are considered to be key players in signal transduction by generating certain phospholipids that are collectively involved in many homeostatic cell functions such as cell proliferation and differentiation, metabolism and immune functions. ${ }^{1-3)}$ Different isoenzymes of this family can be grouped into three classes; class I ( $\mathrm{PI} 3 \mathrm{~K} \alpha, \mathrm{PI} 3 \mathrm{~K} \beta, \mathrm{PI} 3 \mathrm{~K} \delta$ and $\mathrm{PI} 3 \mathrm{~K} \gamma$ ), class II (PI3K-C $2 \alpha, \mathrm{PI} 3 \mathrm{~K}-\mathrm{C} 2 \beta$ and PI3K-C $2 \gamma$ ) and class III (Vps34). ${ }^{4}$

Of these classes, class I PI3Ks is the most studied and it is further subdivided into class IA, comprising three members $(\mathrm{PI} 3 \mathrm{~K} \alpha, \mathrm{PI} 3 \mathrm{~K} \beta, \mathrm{PI} 3 \mathrm{~K} \delta$ ), and class IB with its sole member $\mathrm{PI} 3 \mathrm{~K} \gamma,{ }^{1)}$ differing in their tissue expression levels and their mechanism of activation, class IB PI3K $\gamma$ was found to be predominantly expressed in cells of the hematopoietic system, specifically leukocytes, and is activated through G-protein coupled receptors (GPCRs), unlike Class IA members, such as $\mathrm{PI} 3 \mathrm{~K} \alpha$ and $\mathrm{PI} 3 \mathrm{~K} \beta$ which are ubiquitously expressed in the body and are activated through receptor tyrosine kinases. ${ }^{5}$ Moreover, PI3K $\gamma$ was found to be involved in many inflammatory signaling pathways leading to leukocytic migration and chemotaxis, reactive oxygen species (ROS) production and mast cell degranulation. ${ }^{5,6)}$ On the other hand class II isoenzymes are the least understood and their functions are still ambigious. ${ }^{4-6)}$

The authors declare no conflict of interest.
Thus, it is believed that the design of new inhibitors of PI3K $\gamma$ could present a novel strategy for the treatment of inflammatory diseases. However, such inhibitors should be selective to $\mathrm{PI} 3 \mathrm{~K} \gamma$ in order to minimize the side effects associated with interfering with the function of other PI3K enzymes. In the past few years, numerous selective PI3K $\gamma$ inhibitors have been developed, many of which can be grouped into three chemical classes: thiazolidinones, aminoheterocyclic derivatives and quinazoline derivatives. ${ }^{6,7)}$

Herein, we report the design and synthesis of novel 6-substituted $N^{4}$-acetyl aminoquinazoline derivatives (D) as a hybrid structure obtained from two scaffolds that were previously reported to inhibit $\mathrm{PI} 3 \mathrm{~K} \gamma$, which are quinazolinone derivatives and aminoheterocyclic derivatives (B and $\mathbf{C})^{8,9)}$ $(\mathbf{A})^{7)}$ (Fig. 1). Moreover, two extra series of compounds were synthesized, which are 6-substituted 4-aminoquinazolines (E) and quinazoline-4-ones (F) to test the effect of removing the $N^{4}$-acetyl group on the PI3K $\gamma$ inhibitory effect.

Of the many interactions that have been reported between the active site of PI3K $\gamma$ and previously discovered inhibitors, hydrogen bonding with Val882 in the hinge region is considered the most frequently identified and reported in either co-crystal structures or docking results for most $\mathrm{PI} 3 \mathrm{~K} \gamma$ inhibitors. ${ }^{8-11)}$ Since the design of our aminoquinazoline scaffold was derived from such previous inhibitors, a docking experiment was implemented to test if the Val882 H-bonding is still conserved for our synthesized compounds. Gold software was used for this docking experiment with the crystal structure of PI3K $\gamma$ (PDB code: 3DBS). ${ }^{12)}$ Results showed that most of our synthesized compounds were involved in hydrogen bonding between $\mathrm{N} 1$ of the quinazoline ring and Val882 as well as other $\mathrm{CH}-\pi$ interactions with Ile881, Ile963 and Tyr867 (Fig. 2). These findings suggest a possible activity for our designed compounds which still needs to be confirmed by biological testing.

Synthesis of the quinazoline nucleus was carried out through reflux of 2-amino-5-nitrobenzonitrile 1 with formamide, as shown in Chart 1, to give the 6-nitro-iminoquinazoline derivative 2 . The cyclization reaction was confirmed by the disappearance of the cyano group absorption peak at $2228.6 \mathrm{~cm}^{-1}$ in the IR spectrum. Moreover, its ${ }^{1} \mathrm{H}-\mathrm{NMR}$ spectrum showed two broad singlet peaks at 8.52 and $8.20 \mathrm{ppm}$ corresponding to the protons of the two $-\mathrm{NH}$ groups which would suggest the predominance of the 4-imino rather than the 4-amino quinazoline derivative. Following reduction of the nitro group, different carboxamide or urea derivatives were introduced in position 6 by reaction of quinazoline-4,6diamine 3 with the corresponding acyl chloride or isocyanate,

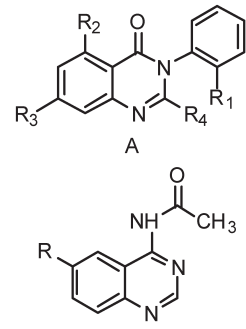

$\mathrm{D}$
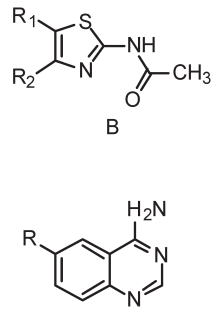

$\mathrm{E}$
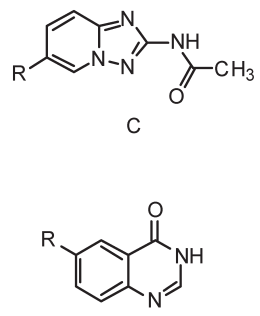

$\mathrm{F}$
Fig. 1. Scaffolds of Previously Reported Inhibitors to PI3K $\gamma(\mathbf{A}-\mathbf{C})$ and Scaffolds of Synthesized Compounds in This Work (D, E) 
respectively. The regioselectivity of this reaction was confirmed by the ${ }^{1} \mathrm{H}-\mathrm{NMR}$ spectrum in which the singlet peak corresponding to the 6-amino group $(5.38 \mathrm{ppm})$ disappeared, while the singlet peak corresponding to the 4-amino group (7.33 ppm) remained. An acetyl moiety was then introduced on the amino group in position 4 using acetic anhydride to give the corresponding acetylated carboxamide or acetylated urea derivatives.

An alternative, parallel scheme was followed for synthesizing other 6-urea or thiourea derivatives of $N^{4}$-acetyl aminoquinazolines (Chart 2), where the 4-imino intermediate 2 was acetylated by reflux in acetic anhydride to give $N$-(6nitroquinazolin-4-yl)acetamide $\mathbf{8}$ followed by reduction of the nitro group using palladium on activated carbon in presence of $\mathrm{H}_{2}$ gas giving the $\mathrm{N}$-(6-aminoquinazolin-4-yl)acetamide 9. It should be noted that a trial was conducted in which the acetylated derivative $\mathbf{8}$ was reduced using stannous chloride, as in the first Chart, but breakage of the acetyl group occurred. Such breakage was evident by the absence of the $\mathrm{CH}_{3}$ peak in the ${ }^{1} \mathrm{H}-\mathrm{NMR}$ spectrum of the reduction product.

Following the reduction step, different side chains were introduced in position 6 of the quinazoline ring by the reaction

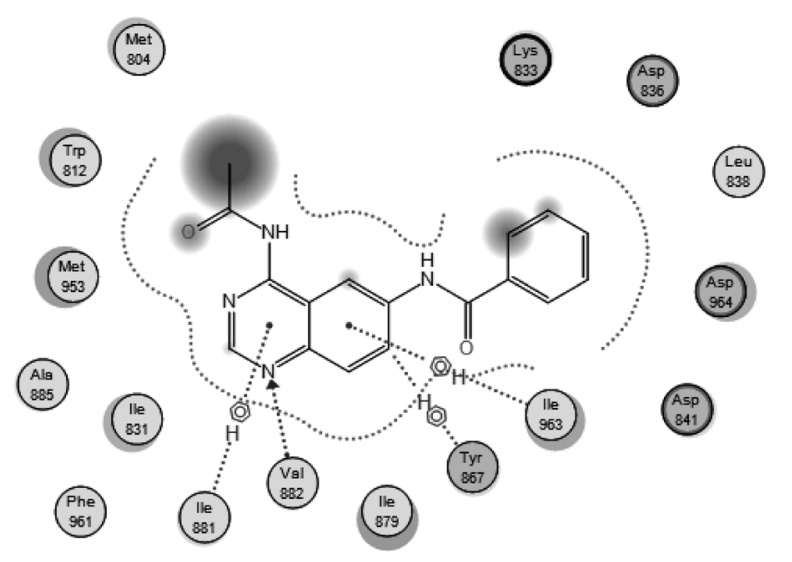

Fig. 2. 2D Ligand Interactions of One of Our Compounds (5a) with $\mathrm{PI} 3 \mathrm{~K} \gamma$

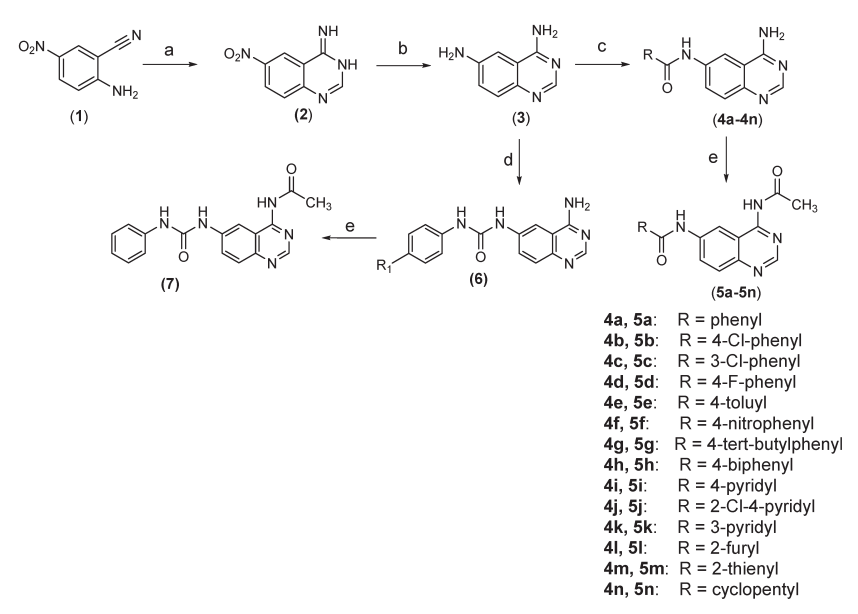

(a) Formamide, reflux, $10 \mathrm{~h}$; (b) $\mathrm{SnCl}_{2} \cdot 2 \mathrm{H}_{2} \mathrm{O} / \mathrm{MeOH}$, reflux, $3 \mathrm{~h}$; (c) $\mathrm{RCOCl}$, $\mathrm{NaHCO}_{3}$, acetone or DMF, $0^{\circ} \mathrm{C}, 2-3 \mathrm{~h}$; (d) Ph-NCO, DMF, $0^{\circ} \mathrm{C}, 2 \mathrm{~h}$; (e) acetic anhydride, reflux, $30 \mathrm{~min}$.

Chart 1

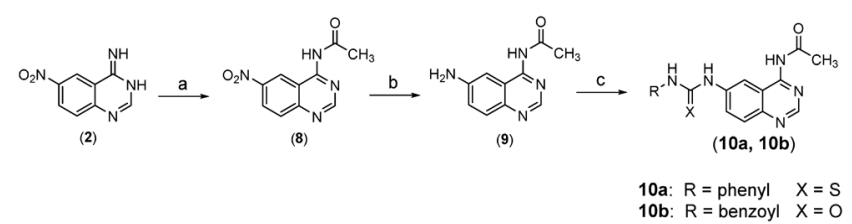

(a) Acetic anhydride, reflux, 30 min; (b) $\mathrm{H}_{2}, \mathrm{Pd} / \mathrm{C}, \mathrm{MeOH}, 3 \mathrm{~h}$; (c) RNCX, DMF, rt, $2-3 \mathrm{~h}$.

Chart 2

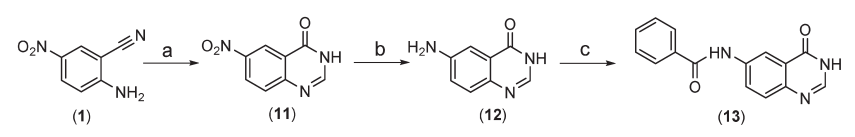

(a) Formic acid, reflux, 6h; (b) $\mathrm{SnCl}_{2} \cdot 2 \mathrm{H}_{2} \mathrm{O} / \mathrm{MeOH}$, reflux, $3 \mathrm{~h}$; (c) $\mathrm{PhCOCl}$, $\mathrm{NaHCO}_{3}$, acetone, $0^{\circ} \mathrm{C}, 3 \mathrm{~h}$.

Chart 3

Table 1. \% Residual Activity of PI3Ks Following Inhibition by 6-Substituted $N^{4}$-Acetyl Aminoquinazoline Derivatives

\begin{tabular}{|c|c|c|c|c|c|c|c|c|}
\hline Compound & $\mathrm{PI} 3 \mathrm{~K} \gamma$ & $\mathrm{PI} 3 \mathrm{~K} \alpha$ & $\mathrm{PI} 3 \mathrm{~K} \beta$ & $\mathrm{PI} 3 \mathrm{~K} \delta$ & $\mathrm{PI} 3 \mathrm{~K}-\mathrm{C} 2 \alpha$ & PI $3 \mathrm{~K}-\mathrm{C} 2 \beta$ & $\mathrm{PI} 3 \mathrm{~K}-\mathrm{C} 2 \gamma$ & Vps34 \\
\hline $5 a$ & 72 & 71 & 89 & 78 & 81 & 87 & 47 & 90 \\
\hline $5 b$ & 85 & 88 & 82 & 84 & 94 & 97 & 83 & 89 \\
\hline $5 c$ & 87 & 85 & 89 & 79 & 95 & 91 & 79 & 96 \\
\hline $5 d$ & 80 & 78 & 78 & 78 & 88 & 92 & 77 & 84 \\
\hline $5 e$ & 83 & 86 & 124 & 81 & 94 & 93 & 45 & 95 \\
\hline $5 f$ & 104 & 87 & 81 & 84 & 92 & 99 & 84 & 90 \\
\hline $5 g$ & 98 & 78 & 70 & 82 & 96 & 94 & 91 & 89 \\
\hline $5 \mathrm{~h}$ & 90 & 87 & 81 & 83 & 91 & 91 & 83 & 96 \\
\hline $5 i$ & 81 & 73 & 69 & 81 & 81 & 96 & 74 & 93 \\
\hline $5 j$ & 85 & 88 & 136 & 85 & 91 & 97 & 63 & 96 \\
\hline $5 \mathrm{k}$ & 93 & 90 & 100 & 85 & 90 & 95 & 83 & 96 \\
\hline 51 & 102 & 86 & 78 & 87 & 93 & 93 & 69 & 99 \\
\hline 7 & 83 & 87 & 76 & 81 & 90 & 89 & 73 & 93 \\
\hline $10 \mathrm{a}$ & 94 & ND & ND & ND & ND & ND & ND & ND \\
\hline $10 \mathrm{~b}$ & 97 & ND & ND & ND & ND & ND & ND & ND \\
\hline 13 & 97 & ND & ND & ND & ND & ND & ND & ND \\
\hline $4 a$ & 89 & ND & ND & ND & ND & ND & ND & ND \\
\hline
\end{tabular}

Residual activity as \% of control. ND: Not determined. 
with the corresponding isocyanates or isothiocyanates to give thiourea or benzoyl urea derivatives $10 \mathbf{a}$ and $\mathbf{b}$.

Aside from the 4-aminoquinazoline derivatives, the quinazoline-4-one nucleus was synthesized by refluxing 2-amino5-nitrobenzonitrile 2 with formic acid to give 6-nitroquinazolin-4-(3H)-one 11 (Chart 3). Cyclization was confirmed similarly to the 4-imino-quinazoline nucleus by the disappearance of the cyano absorption peak from the IR spectrum. The nitro group of compound $\mathbf{1 1}$ was reduced using stannous chloride, followed by acylation of the newly formed amine using the corresponding acid chlorides to give final compounds 13a and $13 b$.

The synthesized compounds were biologically assayed, using the enzymatic ADP-Glo ${ }^{\mathrm{TM}}$ assay, for their inhibitory effect on the eight different PI3K isoforms in order to test their activity as well as their selectivity. Testing was done at a single screening dose of $10 \mu \mathrm{m}$ where the inhibitory activities of the compounds are expressed based on the residual activity as $\%$ of control and are shown in Table 1 .

The major set of tested compounds contained an aryl or heteroaryl group directly linked through an amide linker to position 6 of the quinazoline ring. Based on the residual activity of PI3K $\gamma$ at a single dose of $10 \mu \mathrm{M}$ of our tested compounds.

Compound 5a having an unsubstituted phenyl group showed appreciable inhibition to the isoform PI3K-C $2 \gamma$ when compared to other monosubstituted phenyl derivatives with either a para substituent, particularly as in compounds $\mathbf{5 b}$, d, and $\mathbf{g}$, or a meta substitutent, as in compound $\mathbf{5 c}$. Comparing the effect of different substituents on the phenyl ring, it was found that a $p$-fluoro substituent led to a decrease in activity when compared to the unsubstituted phenyl which suggested that an electon withdrawing group in the para position may lead to a decrease in activity. Moreover, the replacement of the $p$-chloro substituent in $\mathbf{5 b}$ with a less bulky $p$-fluoro in $\mathbf{5 d}$ increased the activity, while replacement with the more bulky $p$-tert-butyl and $p$-nitro in $\mathbf{5 f}$ and $\mathbf{g}$, respectively decreases the activity. This trend suggested that aside from the electronic effect of the substituent, increasing the size of the para substituent would lower the activity as PI3K $\gamma$ inhibitors. In addition, moving the bulky chloro substituent from the para to the meta position in compound $\mathbf{5} \mathbf{c}$ caused no significant change in activity.

On the other hand, the bioisosteric replacement of the phenyl ring with a thiophene ring in compound $\mathbf{5 k}$ led to a significant decrease in activity. This drop in activity was observed with other heteroaryl groups exemplified in either a 6 membered heteroaromatic ring such as pyridine in compounds $\mathbf{5 h}$ and $\mathbf{i}$ or a 5 membered heteroaromatic ring such as furan in compound $\mathbf{5} \mathbf{j}$. In addition, replacing the aromatic ring with an alicyclic ring such as cyclopentane in compound $\mathbf{5 l}$ led to a significant decrease of activity which probably indicates the importance of a $\pi$-electron system in this position. Finally, replacing the amide linkage present between the aryl moiety and the quinazoline ring with a more extended linkage, such as urea or thiourea, led to a decrease in activity. This was proven by comparing the activity of the urea derivatives $(7,10 \mathrm{~b})$ and the thiourea derivative (10a) to their carboxamide analogue 5a. It was also found that the 4-amino- and 4-oxo derivatives (4a, 13, respectively) showed decreased activity when compared to their $N^{4}$ acetyl analogue 5a, which suggested the importance of the acetyl group in this position.
Finally, despite the fact that the tested compounds didn't show appreciable inhibitory effect on $\mathrm{PI} 3 \mathrm{~K} \gamma$ when compared to other isoforms, two compounds (5a, e) showed a significant, inhibitory effect on PI3K-C2 $\gamma$, one of class II PI3Ks. This may be useful for further modification of these two compounds to develop potent, selective inhibitors for this isoform whose function in the body is not yet determined and to which no inhibitors have been reported.

\section{Experimental}

Chemistry All reagents and solvents were of commercial quality and used without further purification. Reactions were carried out under argon whenever inert atmosphere was needed. Flash chromatography was carried out using Interchim Puriflash with stationary phase DAVISIL LC60A 20-45 Micron. NMR data were recorded at ambient temperature on a Bruker Avance 200 at $200 \mathrm{MHz}\left({ }^{1} \mathrm{H}\right)$ or $50 \mathrm{MHz}\left({ }^{13} \mathrm{C}\right)$, a Bruker Ultra Shield 400 at $400 \mathrm{MHz}\left({ }^{1} \mathrm{H}\right)$ or $101 \mathrm{MHz}\left({ }^{13} \mathrm{C}\right)$ or a Bruker DRX-500 at $500 \mathrm{MHz}\left({ }^{1} \mathrm{H}\right)$ or $126 \mathrm{MHz}\left({ }^{13} \mathrm{C}\right)$ using dimethylsulfoxide (DMSO) $-d_{6}$ as the solvent. Chemical shifts $(\delta)$ are reported in ppm relative to the solvent resonance and all coupling constants $(J)$ are given in Hz. Low resolution mass spectral (LR-MS) analysis were performed on a Bruker Esquire 3000 plus (electrospray ionization (ESI)-ion trap) while for all final compounds, High-resolution mass spectral analyses (ESI-time-of-flight (TOF)-MS) were performed on a Bruker MAXIS 4G using ESI as the ionization method. The purity of the final compounds were determined by HPLC on a HPLC Hewlett-Packard HP 1090 Series II liquid chromatograph equipped with a UV diode array detector (DAD) (detection at $230 \mathrm{~nm}$ and $254 \mathrm{~nm}$ ) using a Thermo Betasil C8 column $(150 \mathrm{~mm} \times 4.6 \mathrm{~mm}, \mathrm{dp}=5 \mu \mathrm{m})$ or a ZORBAX Eclipse XDB-C8 column $(150 \mathrm{~mm} \times 4.6 \mathrm{~mm}, \mathrm{dp}=5 \mu \mathrm{m})$, employing a gradient of $0.01 \mathrm{M} \mathrm{KH}_{2} \mathrm{PO}_{4}(\mathrm{pH}=2.3)$ and methanol as the solvent system with a flow rate of $1.5 \mathrm{~mL} / \mathrm{min}$. The purity was determined based on the area under the curve as calculated by Agilent ChemStation Rev. A.09.03. All final compounds were $>95 \%$ pure except for compound 10a having a purity of 94\%. Melting points were determined on a Buchi Melting Point B-540 apparatus and are uncorrected. FT-IR spectra were recorded on Nicolet Avatar 380 spectrometer.

6-Nitroquinazoline-4-(3H)-imine (2) ${ }^{13)} \quad$ To $5 \mathrm{~g}(30 \mathrm{mmol})$ of 2-amino-5-nitrobenzonitrile, $55 \mathrm{~mL}$ of formamide were added and the mixture was refluxed for $10 \mathrm{~h}$ while stirring. The reaction mixture was then poured on ice water yielding a green precipitate which was separated by filtration and washed with acetone to give the pure product 2 . Yield: $70 \%$; mp: $330^{\circ} \mathrm{C}$; ${ }^{1} \mathrm{H}-\mathrm{NMR}\left(500 \mathrm{MHz}, \mathrm{DMSO}-d_{6}\right) \delta: 9.33-9.30(\mathrm{~m}, 1 \mathrm{H})$, 8.55 (s, 1H), 8.52 (s, 1H), $8.46(\mathrm{dd}, J=9.2,2.5 \mathrm{~Hz}, 1 \mathrm{H}), 8.20$ (s, $1 \mathrm{H}), 7.80(\mathrm{~d}, J=9.2 \mathrm{~Hz}, 1 \mathrm{H}) ;{ }^{13} \mathrm{C}-\mathrm{NMR}\left(126 \mathrm{MHz}, \mathrm{DMSO}-d_{6}\right)$ $\delta: 162.79,158.59,153.19,143.82,128.98,126.41,121.39,113.31$; IR $\left(\mathrm{cm}^{-1}\right): 3274,3084,1952,1682,1618,1587,1510,1482$, $1342,1320,1284,1260,1199,1094$; MS (ESI): $m / z=190.8$ $(\mathrm{M}+\mathrm{H})^{+}$.

Quinazoline-4,6-diamine (3) ${ }^{14)}$ A mixture of $1 \mathrm{~g}(5 \mathrm{mmol})$ of 6-nitroquinazoline-4-(3H)-imine 2 and $6 \mathrm{~g}(25 \mathrm{mmol})$ of $\mathrm{SnCl}_{2} \cdot 2 \mathrm{H}_{2} \mathrm{O}$ in $25 \mathrm{~mL}$ methanol was heated to reflux for $3 \mathrm{~h}$ while stirring under inert atmosphere. The solvent was evaporated then $200 \mathrm{~mL}$ saturated aqueous $\mathrm{NaHCO}_{3}$ solution were added to the residue and left stirring for $10 \mathrm{~min}$. The suspension was then filtered and the aqueous filtrate was collected 
and evaporated. The desired product was then extracted from the residue with $N, N$-dimethylformamide (DMF) and purified using column chromatography (85\% dichloromethane (DCM), $15 \% \quad 0.5 \mathrm{M} \mathrm{NH}$ in methanol solution). Yield: 83\%; mp: 280-283 ${ }^{\circ}$; ${ }^{1} \mathrm{H}-\mathrm{NMR}\left(400 \mathrm{MHz}, \mathrm{DMSO}-d_{6}\right) \delta: 8.14(\mathrm{~s}, 1 \mathrm{H})$, $7.42(\mathrm{~d}, J=8.8 \mathrm{~Hz}, 1 \mathrm{H}), 7.33(\mathrm{~s}, 2 \mathrm{H}), 7.16(\mathrm{~d}, J=7.6 \mathrm{~Hz}, 1 \mathrm{H})$, $7.04(\mathrm{~s}, 1 \mathrm{H}), 5.38(\mathrm{~s}, 2 \mathrm{H}) ;{ }^{13} \mathrm{C}-\mathrm{NMR}\left(101 \mathrm{MHz}, \mathrm{DMSO}-d_{6}\right) \delta$ : $160.24,150.78,146.54,141.65,127.71,123.45,115.56,101.99$; MS (ESI): $m / z=160.8(\mathrm{M}+\mathrm{H})^{+}$.

General Synthesis of Compounds $4 a$ to $4 \mathrm{l}$ To a mixture of $1 \mathrm{mmol}$ of quinazoline-4,6-diamine 3 and $1.1 \mathrm{mmol}$ of $\mathrm{NaHCO}_{3}, 15 \mathrm{~mL}$ acetone (or DMF for compounds $\mathbf{4 h}$ and $\mathbf{i}$ ) were added. Then, $1.1 \mathrm{mmol}$ of the corresponding acid chloride was dissolved in $1.5 \mathrm{~mL}$ acetone (or DMF for compounds 4h and i) and was added drop wise to the previously prepared mixture while stirring in an ice bath. The reaction was left for $2-3 \mathrm{~h}$. The mixture was then poured on a saturated solution of $\mathrm{NaHCO}_{3}$ to form a precipitate which was separated by filtration and purified using column chromatography to give the pure compounds $\mathbf{4 a}$ to $\mathbf{4 l}$.

$N$-(4-Aminoquinazolin-6-yl)benzamide (4a) Yield: 35\%; mp: $322-323^{\circ} \mathrm{C} ;{ }^{1} \mathrm{H}-\mathrm{NMR} \quad\left(400 \mathrm{MHz}\right.$, DMSO- $\left.d_{6}\right) \quad \delta$ : 10.52 $(\mathrm{s}, 1 \mathrm{H}), 8.55(\mathrm{~s}, 1 \mathrm{H}), 8.35(\mathrm{~s}, 1 \mathrm{H}), 8.02(\mathrm{~d}, J=7.2 \mathrm{~Hz}, 2 \mathrm{H})$, $7.92(\mathrm{~d}, J=8.9 \mathrm{~Hz}, 1 \mathrm{H}), 7.78-7.60(\mathrm{~m}, 4 \mathrm{H}), 7.56(\mathrm{t}, J=7.3 \mathrm{~Hz}$, $2 \mathrm{H}) ;{ }^{13} \mathrm{C}-\mathrm{NMR} \quad\left(101 \mathrm{MHz}, \mathrm{DMSO}-d_{6}\right) \delta$ : 165.47, 161.48, $154.40,146.64,135.80,134.51,131.70,128.44,128.17,127.61$, 114.49, 114.27; high resolution (HR)-MS (ESI-TOF-HR): $(\mathrm{M}+\mathrm{H})^{+}=265.10859$ (Calcd for 265.10339); purity (HPLC): $99.7 \%$

$\mathrm{N}$-(4-Aminoquinazolin-6-yl)-4-chlorobenzamide Yield: 50\%; mp: $343-345^{\circ} \mathrm{C}$; ${ }^{1} \mathrm{H}-\mathrm{NMR}\left(400 \mathrm{MHz}, \mathrm{DMSO}-d_{6}\right)$ $\delta: 10.59$ (s, $1 \mathrm{H}), 8.52$ (s, $1 \mathrm{H}), 8.35(\mathrm{~s}, 1 \mathrm{H}), 8.05$ (d, J=8.3 Hz, $2 \mathrm{H}), 7.90(\mathrm{~d}, J=8.9 \mathrm{~Hz}, 1 \mathrm{H}), 7.68$ (d, $J=9.1 \mathrm{~Hz}, 2 \mathrm{H}), 7.64$ (d, $J=8.4 \mathrm{~Hz}, 3 \mathrm{H}) ;{ }^{13} \mathrm{C}-\mathrm{NMR}\left(101 \mathrm{MHz}, \mathrm{DMSO}-d_{6}\right) \delta: 164.38$, $161.47,154.50,146.77,136.58,135.55,133.19,129.58,128.55$, $128.15, \quad 127.70, \quad 114.65, \quad 114.26$; HR-MS (ESI-TOF-HR): $(\mathrm{M}+\mathrm{H})^{+}=299.06950$ (Calcd for 299.06942); purity (HPLC): $100 \%$.

$\mathrm{N}$-(4-Aminoquinazolin-6-yl)-3-chlorobenzamide

(4c)

Yield: $33 \%$; mp: $299-300{ }^{\circ} \mathrm{C}$; ${ }^{1} \mathrm{H}-\mathrm{NMR}\left(400 \mathrm{MHz}, \mathrm{DMSO}-d_{6}\right)$ $\delta: 10.63(\mathrm{~s}, 1 \mathrm{H}), 8.53(\mathrm{~s}, 1 \mathrm{H}), 8.36(\mathrm{~s}, 1 \mathrm{H}), 8.07(\mathrm{~s}, 1 \mathrm{H}), 7.98$ (d, $J=7.4 \mathrm{~Hz}, 1 \mathrm{H}), 7.92(\mathrm{~d}, J=8.8 \mathrm{~Hz}, 1 \mathrm{H}), 7.70(\mathrm{~d}, J=8.6 \mathrm{~Hz}$, $4 \mathrm{H}), 7.60(\mathrm{t}, J=7.7 \mathrm{~Hz}, 1 \mathrm{H}) ;{ }^{13} \mathrm{C}-\mathrm{NMR}(101 \mathrm{MHz}$, DMSO$\left.d_{6}\right) \delta: 164.03,161.51,154.46,146.63,136.47,135.51,133.29$, $131.54,130.48,128.14,127.61,127.38,126.44,114.64,114.23$; MS (ESI): $m / z=298.8(\mathrm{M}+\mathrm{H})^{+}, 300.8\left[(\mathrm{M}+\mathrm{H})^{+}+2\right]$; purity (HPLC): 97\%.

$N$-(4-Aminoquinazolin-6-yl)-4-fluorobenzamide

(4d)

Yield: $38 \%$; mp: $337-338^{\circ} \mathrm{C}$; ${ }^{1} \mathrm{H}-\mathrm{NMR}$ (400 MHz, DMSO$\left.d_{6}\right) \delta: 10.54(\mathrm{~s}, 1 \mathrm{H}), 8.53(\mathrm{~s}, 1 \mathrm{H}), 8.35(\mathrm{~s}, 1 \mathrm{H}), 8.19-8.02(\mathrm{~m}$, 2H), $7.90(\mathrm{~d}, J=8.7 \mathrm{~Hz}, 1 \mathrm{H}), 7.69(\mathrm{~d}, J=9.0 \mathrm{~Hz}, 3 \mathrm{H}), 7.40(\mathrm{t}$, $J=8.6 \mathrm{~Hz}, 2 \mathrm{H}) ;{ }^{13} \mathrm{C}-\mathrm{NMR}\left(101 \mathrm{MHz}, \mathrm{DMSO}-d_{6}\right) \delta: 164.38$, $164.16\left(\mathrm{~d},{ }^{1} J_{\mathrm{C}-\mathrm{F}}=249.4 \mathrm{~Hz}\right), 161.47,154.46,146.74,135.68$, $130.93\left(\mathrm{~d},{ }^{4} J_{\mathrm{C}-\mathrm{F}}=2.8 \mathrm{~Hz}\right), 130.36\left(\mathrm{~d},{ }^{3} J_{\mathrm{C}-\mathrm{F}}=9.1 \mathrm{~Hz}\right), 128.17$, $127.68,115.40\left(\mathrm{~d},{ }^{2} J_{\mathrm{C}-\mathrm{F}}=21.9 \mathrm{~Hz}\right), 114.59,114.27$; MS (ESI): $m / z=282.8(\mathrm{M}+\mathrm{H})^{+}$; purity (HPLC): $98 \%$.

$N$-(4-Aminoquinazolin-6-yl)-4-methylbenzamide

(4e)

Yield: $30 \%$; mp: $314-316^{\circ} \mathrm{C}$; ${ }^{1} \mathrm{H}-\mathrm{NMR}\left(400 \mathrm{MHz}, \mathrm{DMSO}-d_{6}\right)$ $\delta: 10.43(\mathrm{~s}, 1 \mathrm{H}), 8.53(\mathrm{~s}, 1 \mathrm{H}), 8.34(\mathrm{~s}, 1 \mathrm{H}), 7.94(\mathrm{~d}, J=8.1 \mathrm{~Hz}$, 2H), $7.90(\mathrm{~s}, 1 \mathrm{H}), 7.68(\mathrm{~d}, J=9.1 \mathrm{~Hz}, 3 \mathrm{H}), 7.36(\mathrm{~d}, J=7.8 \mathrm{~Hz}$, 2H), 2.40 (s, 3H); ${ }^{13} \mathrm{C}-\mathrm{NMR}\left(101 \mathrm{MHz}, \mathrm{DMSO}-d_{6}\right) \delta: 165.29$,
$161.45,154.40,146.60,141.78,135.85,131.58,128.99,128.17$, $127.65,127.61,114.45,114.26,20.98$; MS (ESI): $m / z=278.9$ $(\mathrm{M}+\mathrm{H})^{+}$; purity (HPLC): 97.1\%.

$N$-(4-Aminoquinazolin-6-yl)-4-nitrobenzamide (4f) Yield: 71\%; mp: $328-330^{\circ} \mathrm{C}$; ${ }^{1} \mathrm{H}-\mathrm{NMR}\left(400 \mathrm{MHz}, \mathrm{DMSO}-d_{6}\right) \delta: 10.84$ $(\mathrm{s}, 1 \mathrm{H}), 8.56(\mathrm{~s}, 1 \mathrm{H}), 8.40(\mathrm{~d}, J=8.3 \mathrm{~Hz}, 2 \mathrm{H}), 8.37(\mathrm{~s}, 1 \mathrm{H}), 8.25$ (d, $J=8.3 \mathrm{~Hz}, 2 \mathrm{H}), 7.93(\mathrm{~d}, J=8.6 \mathrm{~Hz}, 1 \mathrm{H}), 7.76(\mathrm{~s}, 2 \mathrm{H}), 7.71$ $(\mathrm{d}, J=8.8 \mathrm{~Hz}, 1 \mathrm{H}) ;{ }^{13} \mathrm{C}-\mathrm{NMR}\left(101 \mathrm{MHz}, \mathrm{DMSO}-d_{6}\right) \delta: 163.87$, $161.56,154.48,149.28,146.55,140.14,135.35,129.17,128.17$, 127.56, 123.62, 114.81, 114.20; MS (ESI): $m / z=309.9(\mathrm{M}+\mathrm{H})^{+}$; purity (HPLC): 92.1\%.

$N$-(4-Aminoquinazolin-6-yl)-4-(tert-butyl)benzamide (4g) Yield: $35 \%$; mp: $324-325^{\circ} \mathrm{C}$; ${ }^{1} \mathrm{H}-\mathrm{NMR}\left(400 \mathrm{MHz}, \mathrm{DMSO}-d_{6}\right)$ $\delta: 10.42(\mathrm{~s}, 1 \mathrm{H}), 8.54(\mathrm{~s}, 1 \mathrm{H}), 8.34(\mathrm{~s}, 1 \mathrm{H}), 7.96(\mathrm{~d}, J=8.1 \mathrm{~Hz}$, 2H), $7.90(\mathrm{~d}, J=8.9 \mathrm{~Hz}, 1 \mathrm{H}), 7.68(\mathrm{~d}, J=8.9 \mathrm{~Hz}, 1 \mathrm{H}), 7.64$ (s, 2H), 7.58 (d, $J=8.3 \mathrm{~Hz}, 2 \mathrm{H}), 1.34(\mathrm{~s}, 9 \mathrm{H}) ;{ }^{13} \mathrm{C}-\mathrm{NMR}(101 \mathrm{MHz}$, DMSO- $\left.d_{6}\right) \delta$ : 165.41, 161.46, 154.60, 154.37, 146.63, 135.92, $131.79,128.05,127.64,127.50,125.21,114.29,114.24,34.66$, 30.89; MS (ESI): $m / z=320.9(\mathrm{M}+\mathrm{H})^{+}$; purity (HPLC): $98.2 \%$.

$\mathrm{N}$-(4-Aminoquinazolin-6-yl)-2-chloroisonicotinamide (4h) Yield: $70 \%$; mp: $279-280^{\circ} \mathrm{C}$; ${ }^{1} \mathrm{H}-\mathrm{NMR}$ (500 MHz, DMSO$\left.d_{6}\right) \delta: 10.83(\mathrm{~s}, 1 \mathrm{H}), 8.66(\mathrm{dd}, J=5.1,0.7 \mathrm{~Hz}, 1 \mathrm{H}), 8.53(\mathrm{~d}$, $J=2.2 \mathrm{~Hz}, 1 \mathrm{H}), 8.36$ (s, 1H), 8.05 (dd, $J=1.4,0.7 \mathrm{~Hz}, 1 \mathrm{H}), 7.92$ (dd, $J=5.1,1.5 \mathrm{~Hz}, 1 \mathrm{H}), 7.90(\mathrm{dd}, J=9.0,2.3 \mathrm{~Hz}, 1 \mathrm{H}), 7.72$ (s, 2H), $7.70(\mathrm{~s}, 1 \mathrm{H}) ;{ }^{13} \mathrm{C}-\mathrm{NMR}\left(126 \mathrm{MHz}, \mathrm{DMSO}-d_{6}\right) \delta: 162.46$, $161.51,154.73,150.90,150.84,147.02,145.09,134.89,127.94$, 127.89, 122.24, 121.22, 114.79, 114.24; MS (ESI): $m / z=299.8$ $(\mathrm{M}+\mathrm{H})^{+}, 301.8\left[(\mathrm{M}+\mathrm{H})^{+}+2\right]$; purity (HPLC): $100 \%$.

$N$-(4-Aminoquinazolin-6-yl)nicotinamide (4i) Yield: 40\%; mp: $305-307^{\circ} \mathrm{C}$; ${ }^{1} \mathrm{H}-\mathrm{NMR}\left(500 \mathrm{MHz}, \mathrm{DMSO}-d_{6}\right) \delta: 10.70(\mathrm{~s}$, $1 \mathrm{H}), 9.17(\mathrm{dd}, J=2.3,0.7 \mathrm{~Hz}, 1 \mathrm{H}), 8.79(\mathrm{dd}, J=4.8,1.6 \mathrm{~Hz}, 1 \mathrm{H})$, $8.54(\mathrm{~d}, J=2.2 \mathrm{~Hz}, 1 \mathrm{H}), 8.37-8.33(\mathrm{~m}, 2 \mathrm{H}), 7.91$ (dd, $J=8.9$, $2.3 \mathrm{~Hz}, 1 \mathrm{H}), 7.70(\mathrm{~d}, J=8.9 \mathrm{~Hz}, 1 \mathrm{H}), 7.67$ (s, 2H), 7.60 (ddd, $J=7.9,4.8,0.8 \mathrm{~Hz}, 1 \mathrm{H}) ;{ }^{13} \mathrm{C}-\mathrm{NMR}\left(126 \mathrm{MHz}, \mathrm{DMSO}-d_{6}\right) \delta$ : $164.02,161.48,154.59,152.27,148.68,146.89,135.42,135.37$, $130.16,128.02,127.83,123.58,114.59,114.28$; MS (ESI): $m / z=265.8(\mathrm{M}+\mathrm{H})^{+}$; purity (HPLC): $99.2 \%$.

$\mathrm{N}$-(4-Aminoquinazolin-6-yl)furan-2-carboxamide

Yield: $25 \%$; mp: $278-279^{\circ} \mathrm{C}$; ${ }^{1} \mathrm{H}-\mathrm{NMR}\left(400 \mathrm{MHz}, \mathrm{DMSO}-d_{6}\right)$ $\delta: 10.46(\mathrm{~s}, 1 \mathrm{H}), 8.47(\mathrm{~d}, J=1.2 \mathrm{~Hz}, 1 \mathrm{H}), 8.35(\mathrm{~s}, 1 \mathrm{H}), 7.97$ (s, 1H), $7.92(\mathrm{dd}, J=8.9,1.6 \mathrm{~Hz}, 1 \mathrm{H}), 7.68$ (d, $J=9.0 \mathrm{~Hz}, 3 \mathrm{H}), 7.39$ $(\mathrm{d}, J=3.1 \mathrm{~Hz}, 1 \mathrm{H}), 6.73(\mathrm{~d}, J=1.5 \mathrm{~Hz}, 1 \mathrm{H}) ;{ }^{13} \mathrm{C}-\mathrm{NMR}(101 \mathrm{MHz}$, DMSO- $\left.d_{6}\right) \delta$ : 161.46, 156.28, 154.41, 147.36, 146.55, 145.85, $135.10,128.12,127.58,114.86,114.63,114.24,112.16$; MS (ESI): $m / z=254.8(\mathrm{M}+\mathrm{H})^{+}$; purity (HPLC): $100 \%$.

$\mathrm{N}$-(4-Aminoquinazolin-6-yl)thiophene-2-carboxamide $\quad(\mathbf{4 k})$ Yield: $35 \%$; mp: $292-293^{\circ} \mathrm{C}$; ${ }^{1} \mathrm{H}-\mathrm{NMR}\left(400 \mathrm{MHz}, \mathrm{DMSO}-d_{6}\right)$ $\delta: 10.53(\mathrm{~s}, 1 \mathrm{H}), 8.47(\mathrm{~s}, 1 \mathrm{H}), 8.35(\mathrm{~s}, 1 \mathrm{H}), 8.08(\mathrm{~d}, J=2.7 \mathrm{~Hz}$, $1 \mathrm{H}), 7.93-7.84(\mathrm{~m}, 2 \mathrm{H}), 7.69(\mathrm{~d}, J=9.0 \mathrm{~Hz}, 3 \mathrm{H}), 7.26(\mathrm{t}$, $J=3.6 \mathrm{~Hz}, 1 \mathrm{H}) ;{ }^{13} \mathrm{C}-\mathrm{NMR}\left(101 \mathrm{MHz}, \mathrm{DMSO}-d_{6}\right) \delta: 161.47$, $159.95,154.42,146.53,139.66,135.32,132.03,129.22,128.15$, 127.62, 114.67, 114.27; MS (ESI): $m / z=270.8(\mathrm{M}+\mathrm{H})^{+}$; purity (HPLC): 98\%.

$\mathrm{N}$-(4-Aminoquinazolin-6-yl)cyclopentanecarboxamide (4I) Yield: 39\%; mp: $310-311^{\circ} \mathrm{C}$; ${ }^{1} \mathrm{H}-\mathrm{NMR}\left(500 \mathrm{MHz}, \mathrm{DMSO}-d_{6}\right)$ $\delta$ : $10.08(\mathrm{~s}, 1 \mathrm{H}), 8.40(\mathrm{~d}, J=2.1 \mathrm{~Hz}, 1 \mathrm{H}), 8.30(\mathrm{~s}, 1 \mathrm{H}), 7.74$ (dd, $J=8.9,2.3 \mathrm{~Hz}, 1 \mathrm{H}), 7.61$ (d, $J=8.9 \mathrm{~Hz}, 1 \mathrm{H}), 7.56(\mathrm{~s}, 2 \mathrm{H})$, $2.83(\mathrm{p}, J=8.0 \mathrm{~Hz}, 1 \mathrm{H}), 1.91-1.82(\mathrm{~m}, 2 \mathrm{H}), 1.81-1.64(\mathrm{~m}, 4 \mathrm{H})$, $1.63-1.52(\mathrm{~m}, 2 \mathrm{H}) ;{ }^{13} \mathrm{C}-\mathrm{NMR}\left(126 \mathrm{MHz}, \mathrm{DMSO}-d_{6}\right) \delta: 174.38$, $161.35,154.08,146.23,136.15,127.75,126.84,114.36,112.45$, 45.01, 30.05, 25.68; MS (ESI): $m / z=256.9(\mathrm{M}+\mathrm{H})^{+}$; purity 
(HPLC): 99.7\%.

1-(4-Aminoquinazolin-6-yl)-3-phenylurea Derivatives (6) To $1.2 \mathrm{mmol}$ of phenyl isocyanate dissolved in $10 \mathrm{~mL}$ DMF, $1 \mathrm{mmol}$ of quinazoline-4,6-diamine 3 was added and the mixture was then left to stir in an ice bath for $2 \mathrm{~h}$. The reaction mixture was then poured on ice water where a precipitate is formed, separated by filtration and then purified with column chromatography to yield compound 6. Yield: $34 \%$; mp: $318-319^{\circ} \mathrm{C} ;{ }^{1} \mathrm{H}-\mathrm{NMR}\left(400 \mathrm{MHz}, \mathrm{DMSO}-d_{6}\right) \delta: 9.18(\mathrm{~d}$, $J=12.6 \mathrm{~Hz}, 2 \mathrm{H}), 8.29$ (s, $1 \mathrm{H}), 8.12(\mathrm{~s}, 1 \mathrm{H}), 7.85$ (d, $J=7.7 \mathrm{~Hz}$, $1 \mathrm{H}), 7.62(\mathrm{~d}, J=8.7 \mathrm{~Hz}, 3 \mathrm{H}), 7.50$ (d, $J=6.4 \mathrm{~Hz}, 2 \mathrm{H}), 7.29$ (s, $2 \mathrm{H}), 6.97(\mathrm{~s}, 1 \mathrm{H}) ;{ }^{13} \mathrm{C}-\mathrm{NMR}\left(101 \mathrm{MHz}, \mathrm{DMSO}-d_{6}\right) \delta$ : 161.16, $153.65,152.70,145.56,139.69,136.87,128.74,127.86,126.13$, 121.83, 118.17, 114.63, 110.63; MS (ESI): $m / z=279.9(\mathrm{M}+\mathrm{H})^{+}$; purity (HPLC): $99.5 \%$.

General Synthesis of Compounds $5 \mathrm{a}$ to $5 \mathrm{l}$ and 7 To $0.3 \mathrm{mmol}$ of the corresponding compounds $\mathbf{4 a}$ to $\mathbf{4 n}$ and $\mathbf{6}, 3$ to $4 \mathrm{~mL}$ of acetic anhydride were added and the reaction mixture was refluxed for $30 \mathrm{~min}$ while stirring. The product was then filtered, washed with diethyl ether $(3 \times 10 \mathrm{~mL})$ and then re-crystallized from methanol to yield the pure final compounds $\mathbf{5 a}$ to $\mathbf{5 l}$ and 7 .

$N$-(4-Acetamidoquinazolin-6-yl)benzamide (5a) Yield: $62 \%$; mp: $228-229^{\circ} \mathrm{C}$; ${ }^{1} \mathrm{H}-\mathrm{NMR}$ (400 MHz, DMSO-d $)_{6} \quad \delta$ : $10.87(\mathrm{~s}, 1 \mathrm{H}), 10.70(\mathrm{~s}, 1 \mathrm{H}), 8.95(\mathrm{~s}, 1 \mathrm{H}), 8.67$ (s, 1H), 8.29 (d, $J=9.0 \mathrm{~Hz}, 1 \mathrm{H}), 8.02(\mathrm{~d}, J=7.2 \mathrm{~Hz}, 2 \mathrm{H}), 7.98$ (d, $J=9.1 \mathrm{~Hz}$, $1 \mathrm{H}), 7.60(\mathrm{dt}, J=25.0,7.1 \mathrm{~Hz}, 3 \mathrm{H}), 2.32(\mathrm{~s}, 3 \mathrm{H}) ;{ }^{13} \mathrm{C}-\mathrm{NMR}$ $\left(101 \mathrm{MHz}, \mathrm{DMSO}-d_{6}\right) \delta: 170.14,165.90,157.20,152.95,147.99$, $137.70,134.44,131.87,128.81,128.46,128.25,127.72,118.52$, 113.90, 24.04; HR-MS (ESI-TOF-HR): $(\mathrm{M}+\mathrm{Na})^{+}=329.10095$ (Calcd for 329.10090); purity (HPLC): 98.1\%.

$\mathrm{N}$-(4-Acetamidoquinazolin-6-yl)-4-chlorobenzamide $\quad(\mathbf{5 b})$ Yield: $60 \%$; mp: $240-242{ }^{\circ} \mathrm{C}$; ${ }^{1} \mathrm{H}-\mathrm{NMR}\left(400 \mathrm{MHz}, \mathrm{DMSO}-d_{6}\right)$ $\delta$ : $10.88(\mathrm{~s}, 1 \mathrm{H}), 10.75$ (s, 1H), 8.95 (s, 1H), $8.64(\mathrm{~s}, 1 \mathrm{H}), 8.26$ (d, $J=8.9 \mathrm{~Hz}, 1 \mathrm{H}), 8.05(\mathrm{~d}, J=8.2 \mathrm{~Hz}, 2 \mathrm{H}), 7.98(\mathrm{~d}, J=9.0 \mathrm{~Hz}$, $1 \mathrm{H}), 7.65$ (d, $J=8.2 \mathrm{~Hz}, 2 \mathrm{H}), 2.32(\mathrm{~s}, 3 \mathrm{H}) ;{ }^{13} \mathrm{C}-\mathrm{NMR}(101 \mathrm{MHz}$, DMSO- $\left.d_{6}\right) \delta$ : 170.14, 164.78, 157.22, 153.03, 148.05, 137.47, $136.76,133.10,129.69,128.79,128.56,128.30,118.48,114.07$, 24.04; HR-MS (ESI-TOF-HR): $(\mathrm{M}+\mathrm{Na})^{+}=363.06155$ (Calcd for 363.06192); purity (HPLC): 99.4\%.

$\mathrm{N}$-(4-Acetamidoquinazolin-6-yl)-3-chlorobenzamide

Yield: $61 \%$; mp: $248-250{ }^{\circ} \mathrm{C} ;{ }^{1} \mathrm{H}-\mathrm{NMR}\left(400 \mathrm{MHz}, \mathrm{DMSO}-d_{6}\right)$ $\delta$ : $10.88(\mathrm{~s}, 1 \mathrm{H}), 10.78(\mathrm{~s}, 1 \mathrm{H}), 8.96(\mathrm{~s}, 1 \mathrm{H}), 8.63(\mathrm{~s}, 1 \mathrm{H}), 8.27$ (d, $J=8.9 \mathrm{~Hz}, 1 \mathrm{H}), 8.07$ (s, 1H), 7.98 (d, $J=7.7 \mathrm{~Hz}, 2 \mathrm{H}), 7.71$ (d, $J=7.5 \mathrm{~Hz}, 1 \mathrm{H}), 7.61(\mathrm{t}, J=7.7 \mathrm{~Hz}, 1 \mathrm{H}), 2.32(\mathrm{~s}, 3 \mathrm{H}) ;{ }^{13} \mathrm{C}-\mathrm{NMR}$ $\left(101 \mathrm{MHz}, \mathrm{DMSO}-d_{6}\right) \quad \delta: 170.16,164.41,157.24, \quad 153.07$, $148.09,137.38,136.37,133.29,131.70,130.49,128.77,128.33$, 127.46, 126.56, 118.48, 114.14, 24.04; HR-MS (ESI-TOF-HR): $(\mathrm{M}+\mathrm{Na})^{+}=363.06146$ (Calcd for 363.06192); purity (HPLC): $99.6 \%$.

$\mathrm{N}$-(4-Acetamidoquinazolin-6-yl)-4-fluorobenzamide

(5d)

Yield: $51 \%$; mp: $237-239^{\circ} \mathrm{C}$; ${ }^{1} \mathrm{H}-\mathrm{NMR} \quad(400 \mathrm{MHz}, \mathrm{DMSO}-$ $\left.d_{6}\right) \delta: 10.87(\mathrm{~s}, 1 \mathrm{H}), 10.70(\mathrm{~s}, 1 \mathrm{H}), 8.95(\mathrm{~s}, 1 \mathrm{H}), 8.63(\mathrm{~s}$, $1 \mathrm{H}), 8.27(\mathrm{~d}, J=9.1 \mathrm{~Hz}, 1 \mathrm{H}), 8.11(\mathrm{dd}, J=8.4,5.6 \mathrm{~Hz}, 2 \mathrm{H})$, $7.98(\mathrm{~d}, J=9.1 \mathrm{~Hz}, 1 \mathrm{H}), 7.41$ (t, $J=8.7 \mathrm{~Hz}, 2 \mathrm{H}), 2.32$ (s, 3H); ${ }^{13} \mathrm{C}-\mathrm{NMR} \quad\left(101 \mathrm{MHz}, \mathrm{DMSO}-d_{6}\right) \quad \delta: 170.16,164.78,164.25$ $\left(\mathrm{d},{ }^{1} J_{\mathrm{C}-\mathrm{F}}=249.6 \mathrm{~Hz}\right), 157.19,152.99,148.00,137.58,130.83$ $\left(\mathrm{d},{ }^{4} J_{\mathrm{C}-\mathrm{F}}=2.9 \mathrm{~Hz}\right), 130.51 \quad\left(\mathrm{~d},{ }^{3} J_{\mathrm{C}-\mathrm{F}}=9.2 \mathrm{~Hz}\right), 128.82,128.28$, $118.49,115.45\left(\mathrm{~d},{ }^{2} J_{\mathrm{C}-\mathrm{F}}=21.9 \mathrm{~Hz}\right), 113.96,24.04$; HR-MS (ESITOF-HR): $(\mathrm{M}+\mathrm{Na})^{+}=347.09136$ (calcd for 347.09147); purity (HPLC): 98\%.
$N$-(4-Acetamidoquinazolin-6-yl)-4-methylbenzamide

$(5 \mathbf{e})$

Yield: $74 \%$; mp: $232-233^{\circ} \mathrm{C}$; ${ }^{1} \mathrm{H}-\mathrm{NMR}\left(400 \mathrm{MHz}, \mathrm{DMSO}-d_{6}\right)$ $\delta$ : $10.86(\mathrm{~s}, 1 \mathrm{H}), 10.60(\mathrm{~s}, 1 \mathrm{H}), 8.94(\mathrm{~s}, 1 \mathrm{H}), 8.66(\mathrm{~s}, 1 \mathrm{H}), 8.28$ (d, J=8.9 Hz, 1H), 7.98 (s, 1H), 7.94 (d, J=8.3 Hz, 2H), 7.38 (d, $J=7.7 \mathrm{~Hz}, 2 \mathrm{H}), 2.40$ (s, 3H), 2.32 (s, 3H); ${ }^{13} \mathrm{C}-\mathrm{NMR}(101 \mathrm{MHz}$, DMSO- $\left.d_{6}\right) \delta: 170.12,165.68,157.16,152.90,147.93,142.00$, $137.78,131.52$, 128.99, 128.83, 128.21, 127.77, 118.51, 113.80, 24.04, 21.00; HR-MS (ESI-TOF-HR): $(\mathrm{M}+\mathrm{Na})^{+}=343.11665$ (Calcd for 343.11655); purity (HPLC): 99.1\%.

$\mathrm{N}$-(4-Acetamidoquinazolin-6-yl)-4-nitrobenzamide

Yield: $32 \%$; mp: $249-251{ }^{\circ} \mathrm{C}$; ${ }^{1} \mathrm{H}-\mathrm{NMR}\left(400 \mathrm{MHz}, \mathrm{DMSO}-d_{6}\right)$ $\delta$ : $10.99(\mathrm{~s}, 1 \mathrm{H}), 10.89(\mathrm{~s}, 1 \mathrm{H}), 8.97(\mathrm{~s}, 1 \mathrm{H}), 8.65(\mathrm{~s}, 1 \mathrm{H}), 8.41$ (d, $J=7.9 \mathrm{~Hz}, 2 \mathrm{H}), 8.29(\mathrm{~s}, 1 \mathrm{H}), 8.25(\mathrm{~d}, J=8.6 \mathrm{~Hz}, 2 \mathrm{H}), 8.00$ (d, $J=9.0 \mathrm{~Hz}, 1 \mathrm{H}), 2.32$ (s, 3H); ${ }^{13} \mathrm{C}-\mathrm{NMR}$ (101 MHz, DMSO$\left.d_{6}\right) \delta: 170.18,164.26,157.30,153.19,149.35,148.20,140.03$, 137.17, 129.30, 128.74, 128.43, 123.62, 118.47, 114.38, 24.04; HR-MS (ESI-TOF-HR): $\quad(\mathrm{M}+\mathrm{Na})^{+}=374.08586 \quad$ (Calcd for 374.08597); purity (HPLC): 97\%.

$N$-(4-Acetamidoquinazolin-6-yl)-4-tert-butylbenzamide (5g) Yield: $68 \%$; mp: $217-219^{\circ} \mathrm{C}$; ${ }^{1} \mathrm{H}-\mathrm{NMR}\left(400 \mathrm{MHz}, \mathrm{DMSO}-d_{6}\right)$ $\delta$ : $10.86(\mathrm{~s}, 1 \mathrm{H}), 10.61$ (s, 1H), $8.94(\mathrm{~s}, 1 \mathrm{H}), 8.67(\mathrm{~s}, 1 \mathrm{H}), 8.27$ (d, $J=8.9 \mathrm{~Hz}, 1 \mathrm{H}), 8.01-7.92(\mathrm{~m}, 3 \mathrm{H}), 7.59(\mathrm{~d}, J=8.0 \mathrm{~Hz}, 2 \mathrm{H})$, $2.32(\mathrm{~s}, 3 \mathrm{H}), 1.33(\mathrm{~s}, 9 \mathrm{H}) ;{ }^{13} \mathrm{C}-\mathrm{NMR}\left(101 \mathrm{MHz}, \mathrm{DMSO}-d_{6}\right) \delta$ : $170.12,165.82,157.17,154.80,152.89,147.94,137.81,131.73$, $128.77,128.23,127.60,125.23,118.54,113.69,34.69,30.89$, 24.04; HR-MS (ESI-TOF-HR): $(\mathrm{M}+\mathrm{Na})^{+}=385.16366$ (Calcd for 385.16350); purity (HPLC): 99.4\%.

$\mathrm{N}$-(4-Acetamidoquinazolin-6-yl)-2-chloroisonicotinamide (5h) Yield: $62 \%$; mp: $266-267^{\circ} \mathrm{C}$; ${ }^{1} \mathrm{H}-\mathrm{NMR} \quad(400 \mathrm{MHz}$, DMSO- $\left.d_{6}\right) \delta$ : $10.99(\mathrm{~s}, 1 \mathrm{H}), 10.91(\mathrm{~s}, 1 \mathrm{H}), 8.97(\mathrm{~s}, 1 \mathrm{H}), 8.66$ (d, $J=5.0 \mathrm{~Hz}, 1 \mathrm{H}), 8.62(\mathrm{~s}, 1 \mathrm{H}), 8.25(\mathrm{~d}, J=9.0 \mathrm{~Hz}, 1 \mathrm{H}), 8.06$ (s, 1H), 8.00 (d, J=9.1 Hz, 1H), 7.92 (d, $J=4.1 \mathrm{~Hz}, 1 \mathrm{H}), 2.32$ (s, $3 \mathrm{H}) ;{ }^{13} \mathrm{C}-\mathrm{NMR}\left(101 \mathrm{MHz}, \mathrm{DMSO}-d_{6}\right) \delta: 170.18,162.88,157.35$, $153.28,150.89,150.80,148.29,144.98,136.81,128.62,128.50$, 122.29, 121.26, 118.46, 114.52, 24.03.; HR-MS (ESI-TOF-HR): $(\mathrm{M}+\mathrm{Na})^{+}=364.05692$ (Calcd for 364.05717); purity (HPLC): $99.5 \%$.

$\mathrm{N}$-(4-Acetamidoquinazolin-6-yl)nicotinamide (5i) Yield: 59\%; mp: $270-271^{\circ} \mathrm{C} ;{ }^{1} \mathrm{H}-\mathrm{NMR}\left(400 \mathrm{MHz}, \mathrm{DMSO}-d_{6}\right.$ ) $\delta: 10.88$ (s, 2H), $9.17(\mathrm{~s}, 1 \mathrm{H}), 8.96(\mathrm{~s}, 1 \mathrm{H}), 8.80(\mathrm{~d}, J=4.1 \mathrm{~Hz}, 1 \mathrm{H}), 8.65$ $(\mathrm{s}, 1 \mathrm{H}), 8.36(\mathrm{~d}, J=7.8 \mathrm{~Hz}, 1 \mathrm{H}), 8.27(\mathrm{~d}, J=8.8 \mathrm{~Hz}, 1 \mathrm{H}), 7.99$ (d, $J=9.0 \mathrm{~Hz}, 1 \mathrm{H}), 7.61(\mathrm{dd}, J=7.5,5.0 \mathrm{~Hz}, 1 \mathrm{H}), 2.32$ (s, 3H); ${ }^{13} \mathrm{C}-\mathrm{NMR}$ (101 MHz，DMSO- $d_{6}$ ) $\delta$ : 170.16, 164.45, 157.26, $153.10,152.39,148.73,148.10,137.33,135.54,130.13,128.68$, $128.40,123.56,118.50,114.08,24.04$; HR-MS (ESI-TOF-HR): $(\mathrm{M}+\mathrm{Na})^{+}=330.09632$ (Calcd for 330.09615); purity (HPLC): $99.1 \%$.

$N$-(4-Acetamidoquinazolin-6-yl)furan-2-carboxamide $\quad(\mathbf{5 j})$ Yield: $74 \%$; mp: $253-255^{\circ} \mathrm{C}$; ${ }^{1} \mathrm{H}-\mathrm{NMR}$ (400 MHz, DMSO$\left.d_{6}\right) \delta: 10.85(\mathrm{~s}, 1 \mathrm{H}), 10.63(\mathrm{~s}, 1 \mathrm{H}), 8.94(\mathrm{~s}, 1 \mathrm{H}), 8.60(\mathrm{~s}, 1 \mathrm{H})$, $8.29(\mathrm{dd}, J=9.0,1.4 \mathrm{~Hz}, 1 \mathrm{H}), 7.99$ (s, 1H), $7.96(\mathrm{~d}, J=9.1 \mathrm{~Hz}$, $1 \mathrm{H}), 7.43(\mathrm{~d}, J=3.2 \mathrm{~Hz}, 1 \mathrm{H}), 6.75(\mathrm{~d}, J=1.5 \mathrm{~Hz}, 1 \mathrm{H}), 2.32$ $(\mathrm{s}, 3 \mathrm{H}) ;{ }^{13} \mathrm{C}-\mathrm{NMR}\left(101 \mathrm{MHz}, \mathrm{DMSO}-d_{6}\right) \delta$ : 170.14, 157.18, $156.45,152.99,147.96,147.13,146.09,137.08,128.69,128.29$, 118.48, 115.34, 113.99, 112.28, 24.04; HR-MS (ESI-TOF-HR): $(\mathrm{M}+\mathrm{Na})^{+}=319.07979$ (Calcd for 319.08016); purity (HPLC): $98.7 \%$.

$N$-(4-Acetamidoquinazolin-6-yl)thiophene-2-carboxamide (5k) Yield: 65\%; mp: 209-210 ${ }^{\circ} \mathrm{C}$; ${ }^{1} \mathrm{H}-\mathrm{NMR} \quad(400 \mathrm{MHz}$, DMSO- $\left.d_{6}\right) \delta$ : $10.87(\mathrm{~s}, 1 \mathrm{H}), 10.67(\mathrm{~s}, 1 \mathrm{H}), 8.95(\mathrm{~s}, 1 \mathrm{H}), 8.59$ $(\mathrm{s}, 1 \mathrm{H}), 8.25(\mathrm{~d}, J=9.0 \mathrm{~Hz}, 1 \mathrm{H}), 8.11(\mathrm{~d}, J=3.1 \mathrm{~Hz}, 1 \mathrm{H}), 7.98$ 
(d, $J=9.0 \mathrm{~Hz}, 1 \mathrm{H}), 7.92(\mathrm{~d}, J=4.8 \mathrm{~Hz}, 1 \mathrm{H}), 7.27(\mathrm{t}, J=3.9 \mathrm{~Hz}$, $1 \mathrm{H}), 2.32(\mathrm{~s}, 3 \mathrm{H}) ;{ }^{13} \mathrm{C}-\mathrm{NMR}\left(101 \mathrm{MHz}, \mathrm{DMSO}-d_{6}\right) \delta: 170.15$, $160.23,157.16,153.00,147.99,139.41,137.26,132.40,129.61$, 128.72, 128.35, 128.16, 118.47, 113.95, 24.07; HR-MS (ESITOF-HR): $(\mathrm{M}+\mathrm{Na})^{+}=335.05737$ (Calcd for 335.05732); purity (HPLC): 99.1\%.

$\mathrm{N}$-(4-Acetamidoquinazolin-6-yl)cyclopentanecarboxamide (5l) Yield: $61 \%$; mp: $246-248^{\circ} \mathrm{C}$; ${ }^{1} \mathrm{H}-\mathrm{NMR} \quad(400 \mathrm{MHz}$, DMSO- $\left.d_{6}\right) \delta$ : $10.81(\mathrm{~s}, 1 \mathrm{H}), 10.32(\mathrm{~s}, 1 \mathrm{H}), 8.91(\mathrm{~s}, 1 \mathrm{H}), 8.50$ $(\mathrm{s}, 1 \mathrm{H}), 8.06(\mathrm{~d}, J=9.1 \mathrm{~Hz}, 1 \mathrm{H}), 7.91(\mathrm{~d}, J=9.0 \mathrm{~Hz}, 1 \mathrm{H}), 2.85$ (p, $J=7.7 \mathrm{~Hz}, 1 \mathrm{H}), 2.29$ (s, 3H), 1.94-1.81 (m, 2H), 1.81-1.65 $(\mathrm{m}, 4 \mathrm{H}), 1.62-1.51(\mathrm{~m}, 2 \mathrm{H})$; ${ }^{13} \mathrm{C}-\mathrm{NMR}$ (101 MHz, DMSO$\left.d_{6}\right) \delta: 174.87,170.05,157.03,152.60,147.64,137.96,128.33$, 127.79, 118.69, 112.18, 45.27, 30.02, 25.65, 23.99; HR-MS (ESITOF-HR): $(\mathrm{M}+\mathrm{Na})^{+}=321.13216$ (Calcd for 321.13220); purity (HPLC): 98.7\%.

1-(4-Acetamidoquinazolin-6-yl)-3-phenylurea (7) Yield: 47\%; mp: $228-229^{\circ} \mathrm{C} ;{ }^{1} \mathrm{H}-\mathrm{NMR}\left(400 \mathrm{MHz}, \mathrm{DMSO}-d_{6}\right) \delta: 10.83$ $(\mathrm{s}, 1 \mathrm{H}), 9.81(\mathrm{~s}, 1 \mathrm{H}), 9.48(\mathrm{~s}, 1 \mathrm{H}), 8.86(\mathrm{~s}, 1 \mathrm{H}), 8.29(\mathrm{~s}, 1 \mathrm{H})$, $8.00(\mathrm{~s}, 1 \mathrm{H}), 7.90(\mathrm{~s}, 1 \mathrm{H}), 7.51(\mathrm{~s}, 2 \mathrm{H}), 7.29(\mathrm{~s}, 2 \mathrm{H}), 6.98(\mathrm{~s}$, $1 \mathrm{H}), 2.31(\mathrm{~s}, 3 \mathrm{H}) ;{ }^{13} \mathrm{C}-\mathrm{NMR}\left(101 \mathrm{MHz}, \mathrm{DMSO}-d_{6}\right) \delta$ : 170.41, $156.59,152.66,152.04,147.01,139.59,138.70,128.75,128.41$, 127.26, 121.94, 118.82, 118.22, 110.10, 24.13; HR-MS (ESITOF-HR): $(\mathrm{M}+\mathrm{Na})^{+}=344.11170$ (Calcd for 344.11180 ); purity (HPLC): 96\%.

$N$-(6-Nitroquinazolin-4-yl)acetamide (8) ${ }^{15)}$ A suspension of $1 \mathrm{~g}$ of 6-nitroquinazoline-4- $(3 H)$-imine 2 in $15 \mathrm{~mL}$ acetic anhydride was refluxed for $30 \mathrm{~min}$. The product was filtered and washed with diethyl ether $(3 \times 20 \mathrm{~mL})$ to give the pure product 8. Yield: $82 \%$; mp: $271-273^{\circ} \mathrm{C}$; ${ }^{1} \mathrm{H}-\mathrm{NMR}(200 \mathrm{MHz}$, DMSO- $\left.d_{6}\right) \delta: 11.25(\mathrm{~s}, 1 \mathrm{H}), 9.39(\mathrm{~d}, J=2.0 \mathrm{~Hz}, 1 \mathrm{H}), 9.09$ (s, $1 \mathrm{H}), 8.62(\mathrm{dd}, J=9.3,2.5 \mathrm{~Hz}, 1 \mathrm{H}), 8.09(\mathrm{~d}, J=9.1 \mathrm{~Hz}, 1 \mathrm{H})$, $2.40(\mathrm{~s}, 3 \mathrm{H}) ;{ }^{13} \mathrm{C}-\mathrm{NMR}\left(50 \mathrm{MHz}, \mathrm{DMSO}-d_{6}\right) \delta: 170.90,159.18$, $157.50,153.60,145.36,130.31,127.66,122.56,116.18,25.47$; MS (ESI): $m / z=254.8(\mathrm{M}+\mathrm{Na})^{+}$.

$\mathrm{N}$-(6-Aminoquinazolin-4-yl)acetamide (9) To a suspension of $0.3 \mathrm{~g}(1.3 \mathrm{mmol})$ of $N$-(6-nitroquinazolin-4-yl)acetamide 8 in $25 \mathrm{~mL}$ methanol, $30 \mathrm{mg}$ of palladium on activated carbon were added slowly and the reaction mixture was left stirring for $3 \mathrm{~h}$ at room temperature in presence of $\mathrm{H}_{2}$ gas. After completion of the reaction, the mixture was filtered under vacuum and the product was retrieved from the filtrate by evaporation. Yield: $90 \%$; mp: $191-194^{\circ} \mathrm{C} ;{ }^{1} \mathrm{H}-\mathrm{NMR}$ $\left(400 \mathrm{MHz}, \mathrm{DMSO}-d_{6}\right) \delta: 10.48(\mathrm{~s}, 1 \mathrm{H}), 8.64(\mathrm{~s}, 1 \mathrm{H}), 7.66(\mathrm{~d}$, $J=8.9 \mathrm{~Hz}, 1 \mathrm{H}), 7.34(\mathrm{~d}, J=8.8 \mathrm{~Hz}, 1 \mathrm{H}), 6.96(\mathrm{~s}, 1 \mathrm{H}), 5.85$ (s, 2H), $2.24(\mathrm{~s}, 3 \mathrm{H}) ;{ }^{13} \mathrm{C}-\mathrm{NMR}\left(101 \mathrm{MHz}, \mathrm{DMSO}-d_{6}\right) \delta: 169.82$, $154.79,149.22,147.84,144.55,128.58,125.49,120.18,101.56$, 23.92; MS (ESI): $m / z=224.8(\mathrm{M}+\mathrm{Na})^{+}$.

1-(4-Acetamidoquinazolin-6-yl)-3-phenylthiourea (10a) To $1.2 \mathrm{mmol}$ of phenyl isothiocyanate dissolved in $8 \mathrm{~mL} \mathrm{DMF}$, $1 \mathrm{mmol}$ of $\mathrm{N}$-(6-aminoquinazolin-4-yl)acetamide 9 was added and the mixture was left stirring overnight at room temperature. After completion of the reaction, the solution was poured on ice water to form a precipitate which was separated by filtration. The obtained solid was washed with diethyl ether $(3 \times 10 \mathrm{~mL})$ and then re-crystallized from ethyl acetate to yield the pure compound 10a. Yield: $9 \%$; mp: $140-143^{\circ} \mathrm{C}$; ${ }^{1} \mathrm{H}-\mathrm{NMR}\left(400 \mathrm{MHz}, \mathrm{DMSO}-d_{6}\right) \delta: 10.81(\mathrm{~s}, 1 \mathrm{H}), 10.16(\mathrm{~s}, 1 \mathrm{H})$, $10.05(\mathrm{~s}, 1 \mathrm{H}), 8.94(\mathrm{~s}, 1 \mathrm{H}), 8.20(\mathrm{~s}, 1 \mathrm{H}), 8.13(\mathrm{~d}, J=8.4 \mathrm{~Hz}$, $1 \mathrm{H}), 7.91(\mathrm{~d}, J=8.6 \mathrm{~Hz}, 1 \mathrm{H}), 7.49(\mathrm{~d}, J=6.5 \mathrm{~Hz}, 2 \mathrm{H}), 7.36(\mathrm{~s}$, 2H), $7.17(\mathrm{~d}, J=5.9 \mathrm{~Hz}, 1 \mathrm{H}), 2.31(\mathrm{~s}, 3 \mathrm{H}) ;{ }^{13} \mathrm{C}-\mathrm{NMR}(101 \mathrm{MHz}$,
DMSO- $\left.d_{6}\right) \delta: 180.05,170.22,156.86,153.37,148.46,139.14$, $138.18,132.28,128.53,127.62,124.80,123.89,117.81,117.55$, 24.29; HR-MS (ESI-TOF-HR): $(\mathrm{M}+\mathrm{H})^{+}=338.10698$ (Calcd for 338.10701); purity (HPLC): 94.3\%.

1-(4-Acetamidoquinazolin-6-yl)-3-benzoylurea (10b) To $1 \mathrm{mmol}$ of benzoyl isocyanate, $10 \mathrm{~mL}$ of DMF were added followed by the addition of $0.8 \mathrm{mmol}$ of $\mathrm{N}$-(6-aminoquinazolin-4yl)acetamide 9. The reaction mixture was left stirring for $1 \mathrm{~h}$ at room temperature then was poured on ice water to give a white precipitate. After filtration, the product obtained was washed with diethyl ether $(3 \times 10 \mathrm{~mL})$ and was re-crystallized from methanol to give the pure corresponding compounds 10b. Yield: $58 \%$; mp: $262-265^{\circ} \mathrm{C}$; ${ }^{1} \mathrm{H}-\mathrm{NMR} \quad(400 \mathrm{MHz}$, DMSO- $\left.d_{6}\right) \delta$ : $11.20(\mathrm{~s}, 2 \mathrm{H}), 10.82(\mathrm{~s}, 1 \mathrm{H}), 8.92(\mathrm{~s}, 1 \mathrm{H}), 8.40$ (s, 1H), $8.34(\mathrm{~d}, J=9.0 \mathrm{~Hz}, 1 \mathrm{H}), 8.05(\mathrm{~d}, J=7.5 \mathrm{~Hz}, 2 \mathrm{H}), 7.97$ $(\mathrm{d}, J=9.0 \mathrm{~Hz}, 1 \mathrm{H}), 7.68$ (t, $J=7.3 \mathrm{~Hz}, 1 \mathrm{H}), 7.57$ (t, $J=7.6 \mathrm{~Hz}$, 2H), $2.36(\mathrm{~s}, 3 \mathrm{H}) ;{ }^{13} \mathrm{C}-\mathrm{NMR}\left(101 \mathrm{MHz}, \mathrm{DMSO}-d_{6}\right) \delta: 170.36$, $168.85,156.80,152.97,151.29,147.78,136.35,133.15,132.17$, $128.71,128.60,128.30,128.09,117.81,113.09,24.53$; HR-MS (ESI-TOF-HR): $(\mathrm{M}+\mathrm{Na})^{+}=372.10680$ (Calcd for 372.10671); purity (HPLC): $99 \%$.

6-Nitroquinazolin-4-(3H)-one (11) ${ }^{16)}$ To $2 \mathrm{~g}$ of 2-amino5-nitrobenzonitrile $\mathbf{1}, 25 \mathrm{~mL}$ of formic acid were added and the mixture was refluxed for $6 \mathrm{~h}$ while stirring. The reaction mixture was then poured on ice water resulting in the formation of a yellow precipitate which was separated by filtration to give compound $\mathbf{1 1}$.

6-Aminoquinazolin-4-(3H)-one $\quad \mathbf{( 1 2}^{17)}$ A mixture of $1 \mathrm{~g}(5 \mathrm{mmol})$ of 6-nitroquinazolin-4-(3H)-one 11 and $6 \mathrm{~g}$ ( $25 \mathrm{mmol})$ of $\mathrm{SnCl}_{2} \cdot 2 \mathrm{H}_{2} \mathrm{O}$ in $25 \mathrm{~mL}$ methanol was heated to reflux for $3 \mathrm{~h}$ while stirring under argon. The solvent was evaporated then $200 \mathrm{~mL}$ saturated aqueous $\mathrm{NaHCO}_{3}$ solution were added to the residue and left stirring for $10 \mathrm{~min}$. The suspension was then filtered and the aqueous filtrate was collected and evaporated. The product was then extracted from the residue using DMF and purified using column chromatography ( $90 \%$ DCM, $10 \% 0.5 \mathrm{M} \mathrm{NH}_{3}$ in methanol solution) to give the pure compound $\mathbf{1 2}$. Yield: $30 \%$; mp: $310-312^{\circ} \mathrm{C}$; ${ }^{1} \mathrm{H}-\mathrm{NMR}\left(200 \mathrm{MHz}, \mathrm{DMSO}-d_{6}\right) \delta: 11.80(\mathrm{~s}, 1 \mathrm{H}), 7.74(\mathrm{~s}, 1 \mathrm{H})$, $7.36(\mathrm{~d}, J=8.6 \mathrm{~Hz}, 1 \mathrm{H}), 7.17(\mathrm{~d}, J=2.2 \mathrm{~Hz}, 1 \mathrm{H}), 7.05(\mathrm{dd}, J=8.7$, $2.4 \mathrm{~Hz}, 1 \mathrm{H}), 5.59(\mathrm{~s}, 2 \mathrm{H}) ;{ }^{13} \mathrm{C}-\mathrm{NMR}\left(50 \mathrm{MHz}, \mathrm{DMSO}-d_{6}\right) \delta$ : $161.03,148.20,140.63,139.97,128.38,123.99,122.49,106.48$; MS (ESI): $m / z=161.9(\mathrm{M}+\mathrm{H})^{+}$.

$\mathrm{N}$-(4-Oxo-3,4-dihydroquinazolin-6-yl)benzamide (13) To a mixture of $1 \mathrm{mmol}$ of 6-aminoquinazolin-4-(3H)-one $\mathbf{1 2}$ and $1.1 \mathrm{mmol}$ of $\mathrm{NaHCO}_{3}, 15 \mathrm{~mL}$ acetone were added. Then, $1.1 \mathrm{mmol}$ of benzoyl chloride was dissolved in $1.5 \mathrm{~mL}$ acetone and was added drop wise to the previously prepared mixture while stirring in an ice bath. The reaction mixture was stirred for $3 \mathrm{~h}$ at $0^{\circ} \mathrm{C}$. The mixture was then poured on a saturated solution of $\mathrm{NaHCO}_{3}$ to form a precipitate which was separated by filtration and purified using column chromatography to give compound 13. Yield: $47 \%$; mp: $322-323^{\circ} \mathrm{C} ;{ }^{1} \mathrm{H}-\mathrm{NMR}$ $\left(400 \mathrm{MHz}, \mathrm{DMSO}-d_{6}\right) \delta: 12.20(\mathrm{~s}, 1 \mathrm{H}), 10.59(\mathrm{~s}, 1 \mathrm{H}), 8.66$ (s, 1H), $8.19(\mathrm{~d}, J=8.8 \mathrm{~Hz}, 1 \mathrm{H}), 8.02(\mathrm{~d}, J=9.0 \mathrm{~Hz}, 2 \mathrm{H}), 7.99$ (s, 1H), $7.68(\mathrm{~d}, J=8.8 \mathrm{~Hz}, 1 \mathrm{H}), 7.58(\mathrm{dt}, J=25.3,7.1 \mathrm{~Hz}, 3 \mathrm{H})$; ${ }^{13} \mathrm{C}-\mathrm{NMR}$ (101 MHz, DMSO- $d_{6}$ ) $\delta$ : 165.70, 160.60, 144.93, $144.07,137.69,134.56,131.74,128.41,127.69,127.65,127.00$, 122.83, 115.64; HR-MS (ESI-TOF-HR): $(\mathrm{M}+\mathrm{Na})^{+}=288.07449$ (Calcd for 288.07435); purity (HPLC): 99.9\%.

Molecular Modeling GOLD software was used to per- 
form the docking protocol using a co-crystal structure of a $\mathrm{PI} 3 \mathrm{~K} \gamma$ inhibitor with the enzyme (PDB code: $3 \mathrm{DBS}){ }^{12)}$ The binding site residues of $\mathrm{PI} 3 \mathrm{~K} \gamma$ were defined by specifying the crystal structure ligand coordinates and using a cutoff radius of $10 \AA$, with the 'detect cavity' option enabled. The docking protocol was performed using Gold Score as the scoring function and the search efficiency of the genetic algorithm was increased to $125 \%$. Early termination of the docking experiment was enabled when the top three solutions were within $1.5 \AA$ RMSD. The two dimensional (2D) ligand-receptor interactions of these poses were viewed using the "compute ligand interaction" option of MOE.

Acknowledgments The authors are grateful to the staff of Proqinase Company for carrying out the in vitro enzymatic assay for our synthesized compounds.

\section{References}

1) Bader A. G., Kang S., Zhao L., Vogt P. K., Nat. Rev. Cancer, 5, 921-929 (2005).

2) Engelman J. A., Luo J., Cantley L. C., Nat. Rev. Genet., 7, 606-619 (2006).

3) Vivanco I., Sawyers C. L., Nat. Rev. Cancer, 2, 489-501 (2002).

4) Foster F. M., Traer C. J., Abraham S. M., Fry M. J., J. Cell Sci., 116, 3037-3040 (2003).

5) Ito K., Caramori G., Adcock I. M., J. Pharmacol. Exp. Ther., 321, 1-8 (2007).
6) Rückle T., Schwarz M. K., Rommel C., Nat. Rev. Drug Discov., 5, 903-918 (2006).

7) Kuang R. R., Qian F., Li Z., Wei D. Z., Tang Y., Eur. J. Med. Chem., 41, 558-565 (2006)

8) Bell K., Sunose M., Ellard K., Cansfield A., Taylor J., Miller W., Ramsden N., Bergamini G., Neubauer G., Bioorg. Med. Chem. Lett., 22, 5257-5263 (2012).

9) Oka Y., Yabuuchi T., Fujii Y., Ohtake H., Wakahara S., Matsumoto K., Endo M., Tamura Y., Sekiguchi Y., Bioorg. Med. Chem. Lett., 22, 7534-7538 (2012).

10) Venable J. D., Ameriks M. K., Blevitt J. M., Thurmond R. L., FungLeung W.-P., Recent Pat. Inflamm. Allergy Drug Discov., 4, 1-15 (2010).

11) Pomel V., Klicic J., Covini D., Church D. D., Shaw J. P., Roulin K., Burgat-Charvillon F., Valognes D., Camps M., Chabert C., Gillieron C., Françon B., Perrin D., Leroy D., Gretener D., Nichols A., Vitte P. A., Carboni S., Rommel C., Schwarz M. K., Rückle T., J. Med. Chem., 49, 3857-3871 (2006).

12) Bauer M. R., Ibrahim T. M., Vogel S. M., Boeckler F. M., J. Chem. Inf. Model., 53, 1447-1462 (2013).

13) Loidreau Y., Besson T., Tetrahedron, 67, 4852-4857 (2011).

14) Hagan R. L., Hynes J. B., Pimsler M., Kisliuk R. L., Biochem. Pharmacol., 50, 803-809 (1995).

15) Morley J. S., Simpson J. C. E., J. Chem. Soc., 360-366 (1948).

16) Domarkas J., Dudouit F., Williams C., Qiyu Q., Banerjee R., Brahimi F., Jean-Claude B. J., J. Med. Chem., 49, 3544-3552 (2006).

17) Kulkarni S. S., Singh S., Shah J. R., Low W.-K., Talele T. T., Eur. J. Med. Chem., 50, 264-273 (2012). 